this strict Hennigian approach has not been followed. Some innovations have much to commend them; others will generate little but confusion. It is probably a wise move to erect a separate suborder Plesiadapiformes to accommodate Plesiadapis and its apparent relatives, thus emphasizing the these early Tertiary forms and the "primates of modern aspect" (Simons). Indeed, it is by no means certain that the plesiadapiforms exhibit anything other than convergent dental and basicranial similarities to the more obvious primates.

On the other hand, there are several taxonomic changes which could well have been omitted without in the least detracting from the authors' provisional evolutionary hypotheses. Szalay and Delson do not combine all of the primates of modern aspect (their "euprimates") into a formal taxonomic category, despite abundant evidence that this is a monophyletic grouping, so it is difficult to see why other classificatory novelties were required. Two examples illustrate the problems. Firstly, in line with a few other authors, the lemur family Cheirogaleidae has been moved from the superfamily Lemuroidea (containing all the other Malagasy lemurs) to join the Lorisidae in the superfamily Lorisoidea. Szalay's interpretation of certain morphological evidence, which is shared by some but has been disputed by others (and which is in direct conflict with available biochemical evidence), suggests a and lorisids. Whether or not this hypoenormous morphological gulf between recent common ancestry for cheirogaleids

thesis proves to be tenable in the long term, the immediate practical outcome of the taxonomic upheaval is that the term 'lorisoid', which has had a distinct and useful meaning for decades, has been rendered ambiguous. According to which classification one now follows, the word 'lorisoid' may or may not subsume the mouse lemurs and their relatives. Similarly, Szalay and Delson have reduced the marmoset and tamarin group from family level to the tribe Callitrichini and have lumped them together with Callimico and the Cebinae (Cebus + Saimiri + conjectured fossil relatives) into the family Cebidae. This drastic modification is based on Rosenberger's tenuous interpretation of minor dental and cranial features as "shared derived characters" linking marmosets and tamarins to cebines and is once again in conflict with immunological evidence. Henceforth, to the confusion of many involved in research upon marmosets and tamarins, the term 'cebid' might denote either 'all New World monkeys other than marmosets and tamarins' (this being the almost universally accepted meaning to date) or 'marmosets, tarmarins, Callimico and cebines'.

Must we pay the price of such linguistic confusion as provisional hypotheses are generated in the pursuit of a more scientific understanding of primate evolutionary history?

R. D. Martin is Reader in Physical Anthropology at University College, and Visiting Professor in Zoology at Birkbeck College, University of London, UK.

\section{Conformation of saturated heterocycles}

\section{A.R. Katritzky}

The Conformational Analysis of

Heterocyclic Compounds. By Frank Riddell. Pp. 153. (Academic: London and New York, 1980.) $£ 17, \$ 39.50$.

THIS SMALL book will be of considerable help not only to organic and physical chemists, but also to workers in biochemistry and in other biological areas who need to understand something of the conformational analysis of saturated heterocycles. The first two chapters set the scene by summarizing the fundamental concepts on which conformational analysis is based, and then by picking out and emphasizing the differences which characterize the treatment of saturated heterocycles as compared with their carbocyclic analogues.

The remaining two-thirds of the book is organized into five chapters dealing with (1) 4- and 5-membered rings, (2) 6-membered oxygen-containing rings, (3) 6-membered nitrogen-containing rings, (4) 6 -membered rings containing sulphur or phosphorus, and (5) 7-membered and larger rings. To treat this variety of topics within 150 pages, the author must of course be selective: he has rightly chosen to discuss the most important and fundamental ring systems, and thus little attention is paid to polycyclic derivatives. $\mathrm{He}$ has wisely decided to describe, clearly and in some detail, a smaller number of compounds rather than to attempt an encyclopaedic treatment which could have degenerated into mere lists.

The work has obviously been a labour of love; the proof-reading has been carried out excellently, the diagrams are well drawn and clear, and the book has an authoritative ring about it. Further, the subject has not been without its controversial features and on the whole the author deals fairly with these aspects. Organic Chemistry and Dean of the School of Chemical Sciences at the University of East Anglia, UK, but will be taking up a new appointment as Kenan Professor of Organic Chemistry at the University of Florida, Gainesville, in September 1980.
A.R. Katritzky is at present Professor of
Soviet physics

\section{A. J. Leggett}

Soviet Scientific Reviews. Section A: Physics Reviews Vol.1. Edited by I.M. Khalatnikov. Pp.305. (Harwood: Chur, Switzerland, 1979.) Dfl.125, \$67.75.

THIS book is one of a new series which is intended to bring to English-speaking physicists some of the highlights of current research in the Soviet Union. This first volume contains six review articles which concentrate primarily on advances made by Soviet physicists in papers published in 1977 , although reference is made to earlier, and contemporary non-Soviet, work as appropriate. The reviews fall conveniently into three groups of two papers each: Belavin (on instantons) and Zakharov and Manakov (on solitons) concentrate on mathematical developments in field theory, particularly the use of algebraic geometry, and make little if any reference to experiment; the papers by Volovik (superfluid ${ }^{3} \mathrm{He}$ ) and Ivlev (nonequilibrium phenomena in superconductors) are largely theoretical but refer to relevant experiments from time to time; while those by Pokrovskii and Timofeev (exciton condensation in semiconductors) and Borovik-Romanov and co-workers (spin waves in antiferromagnets) are mainly experimental in content.

These reviews are definitely not of the type which could usefully be picked up by a non-specialist as an introduction to the subject in question. They will probably be most useful to those who, while not actively enough involved in a particular area of research to wish to spend a lot of time reading the original Soviet literature, are nevertheless close enough to it to want to keep up with important developments in some detail. For them, this book may play the role as regards Soviet physics that occasional lectures and the 'grapevine' play for much of non-Soviet research. However, although the editor states that he has tried to include "those fields in which Soviet physics is pursuing a direction of its own" it is not clear in most cases that a review covering non-Soviet (or all) work in the area would necessarily be very different in style or emphasis - which perhaps goes to show that the communication gap is not all that serious after all!

The translation is mostly of a high standard, but I hope that in future volumes the series editors will allow more time for proof-reading and also do something about the type-face used; in the present volume the symbols for italic vee and Greek $\mathrm{nu}$ are almost indistinguishable, which leads on occasion (e.g. p.261) to extreme confusion.

A. J. Leggett is Professor of Theoretical Physics at the University of Sussex, Brighton, U.K. 